\title{
Water intoxication in the course of stimulation of labor with oxytocin
}

\author{
Przemyslaw Szadok, Aleksandra Bajorek, Radoslaw Fluder \\ Specialist Hospital of Gynecology and Obstetrics E. Biernacki, Walbrzych, Poland
}

\begin{abstract}
The antidiuretic attribute of oxytocin can cause many side effects. Water intoxication is one of the most serious complications. The authors describe a case of water intoxication with neurological symptoms and severe hyponatraemia in the course of natural labor stimulated by oxytocin in a low-dose regimen.
\end{abstract}

Key words: oxytocin; antidiuretic effect; water intoxication

Ginekologia Polska 2021; 92, 7: 534-535

The use of oxytocin has been indicated in obstetrics to induce or stimulate labor or miscarriage. Apart from having a powerful uterotonic effect, oxytocin is known to possess antidiuretic properties (a hundred-fold weaker than ADH), due to its similarity to vasopressin. This effect is noticeable even at low doses, as early as approximately $10-15$ minutes after administration has been initiated.

We present a case of a primipara whose uterine contractions in the first stage of labor were stimulated by oxytocin using a low-dose regimen for a short period of time $(5 \mathrm{~h})$. Labor complications included water intoxication with severe hyponatremia and an episode of generalized convulsions in both, the mother and the newborn.

A 26-year-old primipara with non-significant medical history was admitted at 40 weeks of gestation due to amniotic fluid leakage and spontaneous irregular uterine contractions. Obstetric examination revealed the following: effacement of the vaginal part of the cervix, soft cervix, dilation of approximately $4.0 \mathrm{~cm}$, with cervical anterior position of +1 , in the axis of the birth canal. The Bishop score was calculated at 11 points. After cardiotocography, a decision was made to administer oxytocin in a low-dose regimen to stimulate uterine contractions. Next, the patient received epidural anesthesia.

To achieve full dilatation, the patient received a total of approx. $20 \mathrm{mLU}$ of oxytocin diluted in $20 \mathrm{~mL} 0.9 \% \mathrm{NaCl}$ within the next 5 hours. She had free access to oral rehydration with clear fluids.

The course of the first stage of labor was uncomplicated and dynamically stable, continuous cardiotocographic supervision with electronic analysis revealed no irregularities. During the second stage, the obstetric examination revealed an abnormal position of the fetal head (posito capitis recta alta). A decision was made to perform an emergency caesarean section.

Spinal anesthesia was performed in a semi-sitting position. After being placed on the operating table, the patient had a tonic-clonic (grand-mal) seizure, with loss of consciousness, salivation and trismus. A Mayo oropharynx tube was used to maintain patent airway (GCS - 10 points, auscultated FHR - about $100 \mathrm{bpm}$ ).

A full-term viable newborn (male, weight: $3500 \mathrm{~g}$, length: $53 \mathrm{~cm}$ ) in average overall condition was delivered (Apgar 6-6-7; cord blood pH 7.07, Na concentration in arterial cord blood $113 \mathrm{mmol} / \mathrm{L}$ (Tab. 1). A seizure occurred at $20 \mathrm{~min}$. of neonatal life, stopped with phenobarbital. An infusion of glucose and sodium bicarbonate was administered, selective hypothermia was induced.

The patient was transferred to an Intensive Care Unit (ICU). Laboratory tests revealed severe hyponatremia (118 mmol/L). Head computed tomography (CT) reveled no focal lesions or signs of intracranial bleeding. Abdominal CT was also normal. Analgosedation, electrolyte supplementation and diuretic therapy were initiated, resulting in gradual resolution of the symptoms and improvement of the laboratory parameters. 


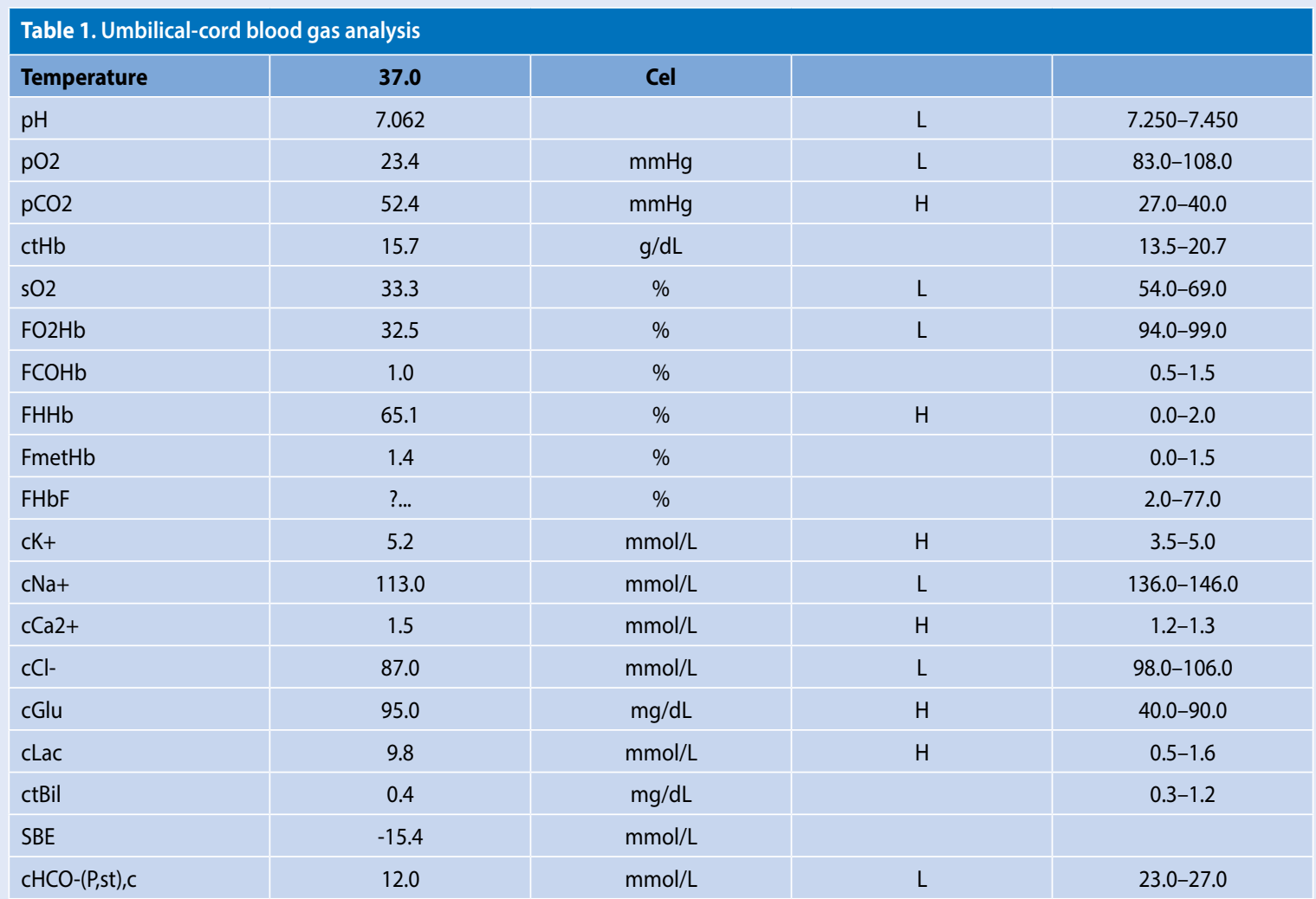

On day five, after cesarean section, the patient was conscious (GCS - 15 points), not requiring electrolyte supplementation, with normal plasma osmolarity and diuresis. During the patient's stay at the ICU, the husband - who had been present during labor - was interviewed and reported that the patient had drunk five liters of low-sodium mineral water during labor. The overall clinical picture and the results of the additional tests lead to the diagnosis of water intoxication caused by the antidiuretic effect of oxytocin.

The problem of water intoxication has been known since the 1960s and the literature offers over a dozen reports of such cases, mainly oxytocin-induced miscarriages or termination of pregnancy up to 24 weeks of gestation. Typically, the protocol was to administer high doses of oxytocin (from 50 to even $140 \mathrm{IU}$ ) in large volumes of the carrier (usually 5-10I $5 \%$ dextrose) over a prolonged period (10-36 hours) [1-4]. The first symptoms included disturbances of consciousness (sometimes challenging to diagnose during labor), tremor, and grand mal convulsions [1, 3]. The problem seems to be significant because it leads to electrolyte disturbance not only in the mother, but also the newborn [3]. In order to minimize the risk of water intoxication, close supervision of the oxytocin dose as well as monitoring of the substances administered in analgesia [2], fluid intake/output, and maternal electrolyte levels [2-5] are recommended. Importantly, despite the availability of prostaglandins [4] used to induce labor or miscarriage and reduced need for oxytocin, the problem of water intoxication has not been eliminated. The rarity of the incidence and the dramatic course of water intoxication make us reflect on the need for an individualized approach to the affected patients.

\section{Conflict of interest}

The authors declare no conflict of interest.

\section{REFERENCES}

1. Lauersen N, Birnbaum S. Water intoxication associated with oxytocin administration during saline-induced abortion. American Journal of Obstetrics and Gynecology. 1975; 121(1): 2-6, doi: 10.1016/0002-9378(75)90965-5.

2. Jensen I, Bruns BJ. Water Intoxication After Oxytocin-Induced Midtrimester Abortion. N Z Med J. 1979; 89(634): 300-302.

3. Ophir E, Solt I, Odeh M, et al. Water intoxication-a dangerous condition in labor and delivery rooms. Obstet Gynecol Surv. 2007; 62(11): 731-738, doi: 10.1097/01.ogx.0000286583.98141.a2, indexed in Pubmed: 17925046.

4. Wang JY, Lin SH, Lin YF, et al. An unforgotten cause of acute hyponatremia: water intoxication due to oxytocin administration in a pregnant woman. Nephron. 2000; 86(3): 342-343, doi: 10.1159/000045791, indexed in Pubmed: 11096293.

5. Feeney JG. Water intoxication and oxytocin. Br Med J (Clin Res Ed). 1982; 285(6337): 243, doi: 10.1136/bmj.285.6337.243, indexed in Pubmed: 6807433. 